\title{
Validez del Cuestionario International Personality Disorder Examination (IPDE) en una muestra de población penitenciaria
}

\author{
E Álvaro-Brun ${ }^{1}$, M Vegue-González ${ }^{2}$
}

${ }^{1}$ C.I.S. Victoria Kent, Madrid

${ }^{2}$ Centro Penitenciario Madrid IV, Navalcarnero, Madrid

\section{RESUMEN}

Objetivo: Conocer los índices validez y establecer el punto de corte más adecuado cuando se utiliza el cuestionario IPDE en una muestra de población penitenciaria.

Material y método: Estudio transversal realizado con una muestra de 100 penados de dos centros penitenciarios de Madrid seleccionados de forma correlativa. Instrumentos de evaluación, cuestionario de datos demográficos, penitenciarios y toxicológicos; y entrevista completa y cuestionario de evaluación IPDE versión DSM-IV. Estudio de sensibilidad y especificidad del cuestionario IPDE con distintos puntos de corte utilizando como "patrón oro" la entrevista IPDE.

Resultados: Utilizando el punto de corte 3 o más respuestas no coincidentes con la esperada, se encuentra baja especificidad $(2,5 \%)$ para la presencia de uno o más trastornos de la personalidad, y baja sensibilidad para los trastornos antisocial $(56,7 \%)$ y límite $(58,8 \%)$ de la personalidad.

Discusión: El cuestionario IPDE tiene escasa utilidad en la población penitenciaria estudiada cuando se aplica con los estándares de referencia habituales, pues el número de falsos positivos que produce es muy elevado. Los mejores índices de validez para identificar uno o más trastornos de la personalidad se obtienen con el punto de corte probable igual a 4 o más respuestas no coincidentes con las esperadas. En cualquier caso el uso del cuestionario IPDE en los reclusos evaluados no aporta beneficios ya que, incluso con el punto de corte habitual de 3 o más respuestas no coincidentes, para los trastornos de personalidad más frecuentes en la muestra estudiada, el antisocial y el límite, se observa que la sensibilidad es baja.

Palabras clave: Trastornos de personalidad, Pruebas de personalidad, Validez de las pruebas, Prisioneros.

\section{VALIDITY OF THE INTERNATIONAL PERSONALITY DISORDER EXAMINATION (IPDE) QUESTIONNAIRE IN A SAMPLE OF PRISON INMATES}

\begin{abstract}
Objective: To discover the validity indices and establish the most adequate cut off point when using the IPDE questionnaire on a sample of prison inmates.

Materials and Methods: A transversal study was carried out on a correlatively selected sample of 100 inmates at two prisons in Madrid. Evaluation instruments, a questionnaire for demographic, prison and toxicological data and a complete interview and IPDE assessment questionnaire (version DSM-IV) were utilised as well as a conditional probability study of the IPDE questionnaire with different cut off points based on the use of the IPDE interview as the "gold standard".

Results: The cut off point of 3 or more non-coincident answers showed low specificity $(2.5 \%)$ for the presence of one or more personality disorders, and low sensitivity to antisocial (56.7\%) and borderline (58.8\%) personality disorders.

Discussion: The IPDE questionnaire of little use amongst the studied prison population when the habitual reference standards were applied due to the very high number of false positives that were produced. The best validity indices for identifying one or more personality disorders are obtained with a probable cut off point being equal to 4 or more answers that do not coincide with those expected.

The IPDE questionnaire was of no great benefit for the inmates in this study because, even when using the habitual cut off point of 3 or more non-coincident questions, sensibility to antisocial and borderline personality disorders, which are the most common PDs amongst the sample group, was found to be low.
\end{abstract}

Key Words: Personality Disorders, Personality tests, Validity of Tests, Prisoners.

Texto recibido: enero 2008

Texto aceptado: mayo 2008 


\section{INTRODUCCIÓN}

Las dificultades metodológicas que plantean los estudios de prevalencia de trastornos de la personalidad producen en ocasiones resultados no coincidentes. Las diferencias que presentan las grandes clasificaciones psiquiátricas y los cambios que se han ido produciendo a lo largo del tiempo en ellas explican que en un conocido estudio comunitario realizado en Estados Unidos con una muestra de 742 sujetos mediante el instrumento diagnóstico IPDE ${ }^{1}$ se encontrara una tasa de prevalencia de trastornos de personalidad del 9,0\% con criterios DSM-IV y del 5,1\% con criterios CIE-10. Pero además, la investigación epidemiológica se dificulta porque la estabilidad de este diagnóstico no es grande, porque resulta complicado disponer de muestras totalmente representativas de la población, y porque se han utilizado una gran variedad de instrumentos de evaluación ${ }^{2}$.

En las revisiones realizadas en reclusos se encuentran en general tasas muy elevadas de trastornos de personalidad ${ }^{3}$, pero no es extraño encontrar resultados dispares, pues las prevalencias en el medio carcelario oscilan entre el $10 \%$ y el $70 \%{ }^{4-7}$.

En las prisiones españolas de Daroca y Zaragoza en el año 1992 se estudió una muestra de 38 reclusos, en todos ellos aparecían criterios diagnósticos de varios trastornos de la personalidad ${ }^{8}$. En otra investigación realizada en 1998 en una muestra de 56 presos del Centro Penitenciario de Monterroso, Lugo, se encontró una prevalencia de trastornos de la personalidad del 91\%9. En el año 2002 en una muestra de 60 internos de la prisión de Zuera, Zaragoza, se observó una prevalencia de trastornos de la personalidad del $30 \%{ }^{10}$. En el año 2005 otro estudio realizado en el Centro Penitenciario Madrid IV y en el Centro de Inserción Social Victoria Kent de Madrid encontró criterios diagnósticos de uno o más trastornos de personalidad en el $60 \%$ de una muestra de 100 internos $^{11}$. Ese mismo año en las prisiones de Daroca y Zaragoza otro estudio realizado con una muestra de 236 internos encontró una prevalencia general de trastornos de personalidad del $69,3 \%{ }^{12}$.

El IPDE es la entrevista semiestructurada utilizada en este trabajo como instrumento de evaluación de los trastornos de la personalidad. Ha sido aprobada por la Organización Mundial de la Salud y se ha traducido al español. Cuenta con versiones DSM-IV y CIE-10, y se utiliza frecuentemente en el ámbito clínico y en investigación al ser una de las entrevistas que presenta mejores propiedades psicométricas. La versión DSM-IV tiene 99 preguntas agrupadas bajo seis encabezamientos: Trabajo, Yo, Relaciones inter- personales, Afectos, Prueba de Realidad y Control de Impulsos. Cada ítem puntúa entre cero y dos puntos, recibe cero puntos cuando la conducta o rasgo a que se refiere la pregunta está ausente o es normal, un punto cuando es exagerado o acentuado y dos puntos cuando es patológico o se cumple el criterio señalado. La corrección puede ser informatizada o manual y facilita un resultado categorial y dimensional. Este instrumento tiene la ventaja de permitir valorar la información obtenida de otras fuentes, como la historia clínica o informadores, cuando estos datos discrepan de los facilitados por el paciente en la entrevista. Requiere que el entrevistador tenga entrenamiento previo en el uso del instrumento y tiene el inconveniente de necesitar mucho tiempo para su aplicación, pues se requieren entre 60 y 90 minutos por término medio para realizar la entrevista.

Para ahorrar tiempo puede usarse el cuestionario IPDE, que es una herramienta de cribaje autoadministrada, con la intención de eliminar aquellos sujetos en los que resulta poco probable que padezcan un trastorno de personalidad, pues aunque este cuestionario genera un número considerable de falsos positivos es de esperar que produzca pocos falsos negativos en relación a la entrevista IPDE. Esto no significa que los dos instrumentos, entrevista y cuestionario, sean intercambiables, ya que el cuestionario IPDE es un instrumento de cribado que no debe ser usado para realizar diagnósticos psiquiátricos en ninguna circunstancia. Este cuestionario en la versión DSM-IV tiene 77 preguntas con dos posibles respuestas, verdadero o falso, y se puede aplicar y corregir mediante un programa informático bajo entorno Windows. El resultado es sólo categorial pues no puede utilizarse para calcular puntuaciones dimensionales.

Este trabajo tiene como objetivo general estudiar la validez de criterio del cuestionario IPDE cuando se utiliza en una muestra de población reclusa, pues en el mismo se compara un instrumento, el cuestionario IPDE, con una prueba diagnóstica que por consenso se considera patrón oro, la entrevista IPDE ${ }^{13-14}$. La validez de criterio puede ser concurrente o predictiva, determinándose en este caso la validez de criterio predictiva, pues los dos instrumentos no se utilizan a la vez, sino que el instrumento que se evalúa, el cuestionario IPDE, se aplica con anterioridad al que sirve de patrón oro. Cuando, como sucede en este caso, se utilizan diagnósticos categoriales y en consecuencia se obtienen variables dicotómicas el estudio de validez se realiza determinando la sensibilidad y especificidad de la prueba.

Los objetivos específicos del estudio son dos. En primer lugar, conocer los índices de validez del cues- 
tionario IPDE en una muestra penitenciaria. En segundo lugar establecer en base a ellos un punto de corte adecuado cuando se utiliza este instrumento en el medio carcelario.

\section{MATERIAL Y MÉTODO}

Se realiza un estudio observacional durante el año 2005 con una muestra formada por 100 penados seleccionados de forma correlativa a medida que se practica una entrevista médica inicial en dos Centros Penitenciarios de Madrid, el Centro Penitenciario Madrid IV, Navalcarnero, y el Centro de Inserción Social Victoria Kent. El perfil de estas personas corresponde a un interno varón, de nacionalidad española, con edad entre 30 y 40 años, soltero, que convive con su familia de origen, con estudios primarios, con escasa cualificación y experiencia profesional, que refiere antecedentes de conducta agresiva, y que cumple más de 5 años de condena por delitos contra la propiedad o contra la Salud Pública.

Se aplicaron tres criterios de inclusión. Primero, rango de edad entre 18 y 65 años. Segundo, situación de estabilidad, permanencia en cualquier centro penitenciario al menos durante 30 días antes del momento de la entrevista. Tercero, situación de abstinencia, ausencia de datos indicativos de consumo de drogas al menos durante 30 días antes del momento de la entrevista. Los criterios de exclusión fueron también tres. Primero, la negativa a colaborar, segundo la dificultad para comprender las preguntas, y tercero la presencia de trastornos psiquiátricos que puedan distorsionar la correcta evaluación o la validez del cuestionario.
En el estudio se utiliza un cuestionario de datos demográficos, penitenciarios y toxicológicos diseñado para este trabajo junto al IPDE, tanto la entrevista IPDE completa en su versión DSM-IV, como el cuestionario de evaluación IPDE.

Este estudio respeta la intimidad de los sujetos, el derecho al anonimato, la voluntariedad en la participación, el derecho a la retirada y el derecho a conocer los objetivos y los procedimientos utilizados en el mismo por parte de los participantes. Los sujetos firmaron un documento de consentimiento informado después de recibir suficiente información por parte de los entrevistadores. La investigación cumple las condiciones fijadas por el Gabinete Técnico de la $\mathrm{Di}$ rección General de Instituciones Penitenciarias para la autorización previa del estudio.

Para cumplimentar el cuestionario IPDE se utiliza el programa informático que acompaña al manual IPDE, este programa permite la corrección automática del cuestionario, y también de la entrevista IPDE después de introducir las puntuaciones en la plantilla correspondiente. El análisis estadístico se realiza con el paquete SPSS (Statistical Package for the Social Sciences) para Windows versión 13.0.

Para estudiar la validez del cuestionario IPDE en comparación con su patrón oro, la entrevista IPDE versión DSM-IV completa, se estudió la sensibilidad, especificidad y el valor predictivo positivo y negativo para distintos puntos de corte.

\section{RESULTADOS}

Con la entrevista IPDE versión DSM-IV presentan criterios diagnósticos de uno o más trastornos de

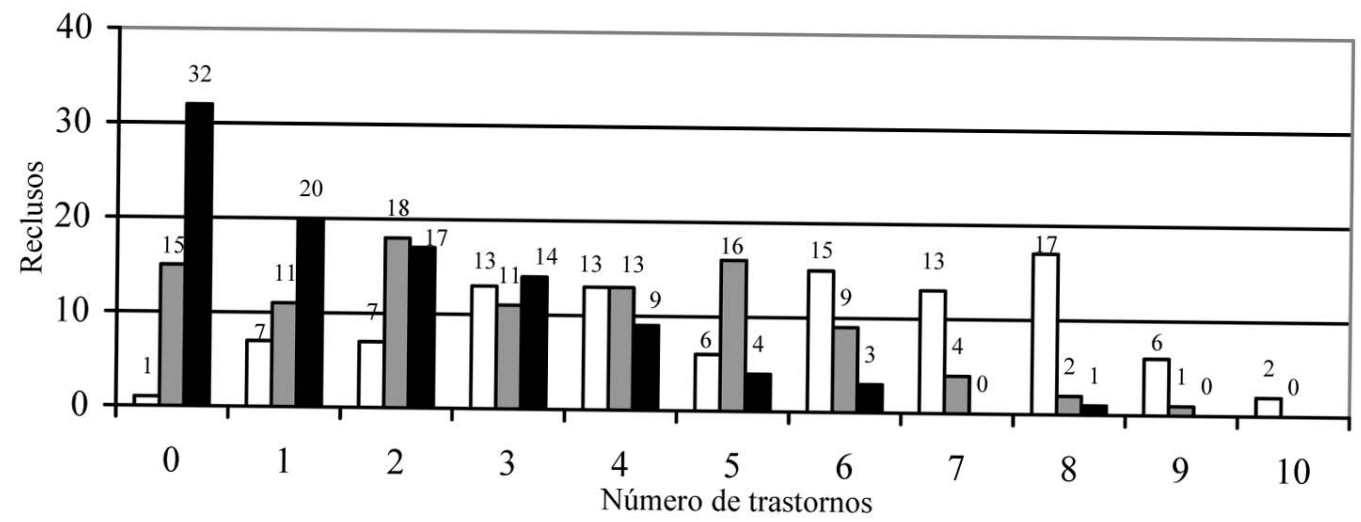

$\square$ Punto corte $3 \square$ Punto de corte $4 \boldsymbol{\square}$ Punto de corte 5

Figura I. Número de trastornos de personalidad probables que presentan los sujetos estudiados con el cuestionario IPDE-DSM-IV para distintos puntos de corte (3, 4 ó 5 respuestas no coincidentes con las esperadas). 
la personalidad el $60 \%$ de los sujetos evaluados, diagnosticando en total 82 trastornos de personalidad. El $40 \%$ no presenta ningún trastorno de la personalidad, el $47 \%$ presenta diagnóstico de un trastorno, el $7 \%$ dos trastornos, el $4 \%$ tres trastornos, el $1 \%$ cuatro trastornos y el $1 \%$ cinco trastornos de la personalidad.

Con el cuestionario IPDE, cuando se utiliza para la corrección el estándar de referencia habitual de tres o más respuestas no coincidentes con las esperadas, el 99\% de los reclusos presenta probablemente algún trastorno de la personalidad, que para su diagnóstico tiene que confirmarse posteriormente con la entrevista IPDE, con este criterio se diagnostican 533 trastornos de personalidad probables entre los reclusos evaluados, con rango entre 0 y 10 trastornos. Aplicando un punto de corte de cuatro o más respuestas no coincidentes con las esperadas, el $85 \%$ de los sujetos presenta probablemente algún trastorno de la personalidad, identificando en total 328 probables trastornos de la personalidad. Cuando se utiliza en la corrección un punto de corte de cinco o más respuestas no coincidentes, el $68 \%$ de los reclusos evaluados con el cuestionario IPDE presenta uno o más posibles trastornos de la personalidad y en total se identifican 159 posibles trastornos de personalidad (Figura I).

En la Tabla I se presenta el resultado de aplicar el cuestionario IPDE-DSM-IV con diferentes puntos de corte distinguiendo para cada uno de ellos los casos en que se confirma o no el diagnóstico de trastorno de la personalidad con la entrevista IPDE.

\begin{tabular}{lccc}
\hline Cuestionario IPDE & \multicolumn{3}{c}{ Entrevista IPDE } \\
& TP & No TP & Total \\
\hline Probable $=3$ o más respuestas no coincidentes \\
Probable & 60 & 39 & 99 \\
No probable & 0 & 1 & 1 \\
\hline Probable $=4$ o más respuestas no coincidentes \\
$\quad$ Probable & 58 & 27 & 85 \\
$\quad$ No probable & 2 & 13 & 15 \\
\hline Probable $=5$ o más respuestas no coincidentes \\
$\quad$ Probable & 50 & 18 & 68 \\
$\quad$ No probable & 10 & 22 & 32 \\
\hline Total & 60 & 40 & 100 \\
\hline
\end{tabular}

Tabla I. Trastornos de la personalidad probables identificados con el Cuestionario IPDE v.s. trastornos de personalidad diagnosticados con la Entrevista IPDE (TP = Trastorno de personalidad).

La Tabla II resume el estudio de probabilidad condicional considerando "patrón oro" el resultado de la entrevista IPDE módulo DSM-IV, señalando los índices de validez del cuestionario IPDE-DSM-IV para los trastornos de la personalidad en general y de forma independiente para los trastornos de personalidad que se identificaron más frecuentemente en la población penitenciaria evaluada.

\section{DISCUSIÓN}

El cuestionario de evaluación IPDE sólo se utiliza para identificar sujetos con alta probabilidad de presentar trastornos de la personalidad ya que no debe usarse para realizar diagnósticos psiquiátricos, pero al aplicarlo como prueba de cribaje, el estudio de validez de este instrumento indica que tiene escasa utilidad en la población reclusa estudiada cuando se aplica con los estándares de referencia habituales, pues el número de falsos positivos que produce es muy elevado al presentar una especificidad muy baja, 2,5\%. Los mejores índices de validez del cuestionario para identificar trastornos de la personalidad en general se obtienen con un punto de corte para trastorno probable de 4 o más respuestas no coincidentes con las esperadas, aun así la especificidad para identificar uno o más trastornos de la personalidad continúa siendo baja, $32,5 \%$, pero no es posible tomar el punto de corte mayor, de 5 o más respuestas no coincidentes con las esperadas, pues en ese caso la sensibilidad baja hasta el $83,3 \%$, lo que resulta inadecuado al tratarse de una prueba de cribaje ya que el principal requisito de este tipo de instrumentos es precisamente que estén dotados de elevada sensibilidad, lo que en la práctica se traduce en la ausencia de falsos negativos.

En cualquier caso el uso del cuestionario IPDE en los reclusos evaluados no aporta beneficios ya que incluso con el punto de corte habitual de 3 o más respuestas no coincidentes para los trastornos de personalidad más frecuentes en la muestra estudiada, el antisocial y el límite, se observa que la sensibilidad es baja, 56,7 y $58,8 \%$ respectivamente.

Estos resultados no confirman los hallazgos de San Narciso ${ }^{15}$, que estudió la validez del cuestionario IP$\mathrm{DE}$ en usuarios de recursos para drogodependientes concluyendo que esta herramienta resulta útil en la evaluación de los trastornos de la personalidad en esa población. Al comparar los índices de validez con el punto de corte habitual de 3 o más respuestas no coincidentes en ambas subpoblaciones, la sensibilidad para los trastornos antisocial y límite es claramente mayor entre los drogodependientes, $88 \%$ para el trastorno antisocial y $100 \%$ para el trastorno límite. 


\begin{tabular}{|c|c|c|c|c|}
\hline Trastornos de Personalidad & Sensibilidad & Especificidad & VPP & VPV \\
\hline \multicolumn{5}{|l|}{ Cualquier trastorno de personalidad } \\
\hline Probable $=3$ o más respuestas no coincidentes & $100 \%$ & $2,5 \%$ & $60,6 \%$ & $100 \%$ \\
\hline Probable $=4$ o más respuestas no coincidentes & $96,7 \%$ & $32,5 \%$ & $68,2 \%$ & $86,7 \%$ \\
\hline Probable $=5$ o más respuestas no coincidentes & $83,3 \%$ & $55 \%$ & $73,5 \%$ & $68,8 \%$ \\
\hline \multicolumn{5}{|l|}{ Trastorno paranoide } \\
\hline Probable $=3$ o más respuestas no coincidentes & $91,7 \%$ & $54,5 \%$ & $21,6 \%$ & $98,0 \%$ \\
\hline Probable $=4$ o más respuestas no coincidentes & $91,7 \%$ & $80,7 \%$ & $39,3 \%$ & $98,6 \%$ \\
\hline Probable $=5$ o más respuestas no coincidentes & $33,3 \%$ & $90,9 \%$ & $33,3 \%$ & $90,9 \%$ \\
\hline \multicolumn{5}{|l|}{ Trastorno antisocial } \\
\hline Probable $=3$ o más respuestas no coincidentes & $56,7 \%$ & $52,9 \%$ & $34 \%$ & $74 \%$ \\
\hline Probable $=4$ o más respuestas no coincidentes & $36,7 \%$ & $75,7 \%$ & $39,3 \%$ & $73,6 \%$ \\
\hline Probable $=5$ o más respuestas no coincidentes & $16,7 \%$ & $90 \%$ & $41,7 \%$ & $71,6 \%$ \\
\hline \multicolumn{5}{|l|}{ Trastorno límite } \\
\hline Probable $=3$ o más respuestas no coincidentes & $58,8 \%$ & $63,9 \%$ & $25 \%$ & $88,3 \%$ \\
\hline Probable $=4$ o más respuestas no coincidentes & $35,3 \%$ & $84,3 \%$ & $31,6 \%$ & $86,4 \%$ \\
\hline Probable $=5$ o más respuestas no coincidentes & $33,3 \%$ & $85,2 \%$ & $23,5 \%$ & $90,4 \%$ \\
\hline \multicolumn{5}{|l|}{ Trastorno histriónico } \\
\hline Probable $=3$ o más respuestas no coincidentes & $100 \%$ & $41,8 \%$ & $3,4 \%$ & $100 \%$ \\
\hline Probable $=4$ o más respuestas no coincidentes & $100 \%$ & $67,3 \%$ & $5,9 \%$ & $100 \%$ \\
\hline Probable $=5$ o más respuestas no coincidentes & $14,3 \%$ & $100 \%$ & $100 \%$ & $87,7 \%$ \\
\hline \multicolumn{5}{|l|}{ Trastorno por evitación } \\
\hline Probable $=3$ o más respuestas no coincidentes & $75 \%$ & $47,9 \%$ & $5,7 \%$ & $97,9 \%$ \\
\hline Probable $=4$ o más respuestas no coincidentes & $50 \%$ & $67,7 \%$ & $5,9 \%$ & $97,0 \%$ \\
\hline Probable $=5$ o más respuestas no coincidentes & $5 \%$ & $96,2 \%$ & $25 \%$ & $80,2 \%$ \\
\hline \multicolumn{5}{|l|}{ Trastorno obsesivo-compulsivo } \\
\hline Probable $=3$ o más respuestas no coincidentes & $66,7 \%$ & $35 \%$ & $3,1 \%$ & $97,1 \%$ \\
\hline Probable $=4$ o más respuestas no coincidentes & $33,3 \%$ & $48,4 \%$ & $2 \%$ & $96,1 \%$ \\
\hline Probable $=5$ o más respuestas no coincidentes & $0 \%$ & $95,4 \%$ & $0 \%$ & $64,9 \%$ \\
\hline
\end{tabular}

Tabla II. Índices de validez del cuestionario IPDE-DSM-IV para identificar trastornos de personalidad en porcentaje ("Patrón oro" entrevista IPDE módulo DSM-IV, VPP = Valor predictivo positivo; VPN = Valor predictivo negativo)

Los instrumentos de cribado y la mayoría de los instrumentos autoaplicados se caracterizan por presentar mayor sensibilidad que las entrevistas semiestructuradas, llegando incluso a plantear algún autor de forma general que los cuestionarios autoaplicados no serían adecuados para hacer diagnósticos de trastornos de la personalidad al producir mayores tasas de falsos positivos ${ }^{16}$.

La escasa sensibilidad encontrada para algunos trastornos de la personalidad en la muestra penitenciaria estudiada puede ser atribuida a varios motivos. Se ha argumentado que interrogar al paciente acerca de cuestiones directamente relacionadas con los criterios diagnósticos puede ser adecuado para estudiar el Eje I, pero no para evaluar el Eje $\mathrm{II}^{2}$. Por otro lado hay que considerar que el IPDE es, esencialmente, un instrumento autodescriptivo, $y$ asume que una persona es capaz de dar una descripción válida de las alteraciones de su personalidad, sin embargo es posible que un individuo pueda no ser consciente de alguna de sus características por falta de introspección o autoconocimiento ${ }^{1}$. Además las dificultades para el recuerdo y la elevada frecuencia de trastornos comórbidos del Eje I entre la población reclusa pueden afectar la exactitud de las respuestas facilitadas.

Pero también hay que considerar que el sujeto puede disimular rasgos de personalidad indeseables como sucede precisamente en el caso de los trastornos antisocial y límite, especialmente cuando son evaluados en prisión, ya que el conocimiento de determinados comportamientos puede resultar contrario a sus intereses, especialmente cuando esa información se facilita y puede ser usada por el personal penitenciario. Este problema se resuelve cuando se utiliza la 
entrevista IPDE ya que permite tomar en consideración, además de las respuestas del sujeto, el juicio del explorador y la información adicional de diversas fuentes.

A la luz de los datos de este estudio se desprende que resulta aconsejable utilizar en el medio penitenciario directamente la entrevista IPDE y no usar previamente el cuestionario de cribaje, pues la falta de especificidad observada limita el posible ahorro de tiempo, pero sobre todo porque la falta de sensibilidad puede hacer que pasen inadvertidos algunos trastornos de personalidad que presentan elevada prevalencia en el medio carcelario.

\section{BIBLIOGRAFÍA}

1. I.P.D.E. Examen internacional de los trastornos de la personalidad. Organización Mundial de la Salud. Madrid: Meditor, 1996.

2. Pérez A. Epidemiología. En: Roca M (coordinador). Trastornos de personalidad. Barcelona: Psiquiatría Editores, 2004.

3. Fazel S, Danesh J. Serious mental disorder in 23.000 prisoners: A systematic review of 62 surveys. The Lancet. 2002; 359: 545-551.

4. Rotter M, Way B, Steinbacher M, Sawyer D, Smith H. Personality disorders in prison: aren't they all antisocial? Psychiatr Q 2002; 73(4): 337-49.

5. Brooke D, Taylor C, Gunn J, Maden A. Point prevalence of mental disorder in unconvicted male prisoners in England and Wales. BMJ 1996; 313: 1524-1527.

6. Widiger TA, Cadoret R, Hare R et al. DSM-IV antisocial personality disorder field trial. Act Psychiatr Scand 1996; 105: 3-16.

7. Brinded PMJ, Mulder RT, Stevens I, Fairley N, Malcolm F. The Christchurch prisons psychiatric epidemiology study: Personality disorders assessment in a prison population. Criminal Behaviour \& Mental Health 1999; 9: 413-22.

8. Rubio V, Varona J, Muro C, Valls E, Bosqued M, Granada JM, et al. Trastornos de personalidad en población penitenciaria. Actas del I Congreso
Nacional de la Sociedad Española de Psiquiatría Legal. Zaragoza, 1992; 295-303.

9. Riesco Y, Pérez A, Rubio V, Izquierdo JA, Sánchez S, Santos JM, Carrasco JL. Evaluación de los trastornos de la personalidad en una población penal con el IPDE y el MMPI. Actas Luso-Esp Neurol. Psiquiatr. 1998; 26(3): 151-4.

10. Arroyo JM. Los trastornos de personalidad en reclusos como factor de distorsión del clima social de la prisión. IV Congreso Nacional de trastornos de la personalidad. Bilbao, 2002.

11. Álvaro E. Evaluación de la psicopatía en una muestra de reclusos en régimen abierto con la Psychopathy Chechklist de Hare. Madrid: Ministerio del Interior, 2007.

12. López R, Lafuente O, García JL. Del mito de Narciso a los trastornos de la personalidad en las cárceles aragonesas: Una aproximación al perfil de estos desórdenes en las personas privadas de libertad. Rev Esp Sanid Penit 2007; 9: 28-40.

13. Pita S, Pertegas S. Pruebas diagnósticas. Cad Aten Primaria 2003; 10: 120-124.

14. Ochoa C, González de Dios J, Buñuel JC. Evaluación de artículos científicos sobre pruebas diagnósticas, Evid Pediatr. 2007; 3:24.

15. San Narciso GI, Gutiérrez E, Saiz PA, González MP, Bascarán MT, Bobes J. Evaluación de trastornos de la personalidad en pacientes heroinómanos mediante el Internacional Personality Disorders Examination (IPDE). Adicciones, 2000; 12(1): 43-56.

16. Loranger AW. Are current self-report and interview measures adequate for epiedemiological studies of personality disorders? J Person Disord 1992; 6: 313-25.

\section{CORRESPONDENCIA}

Enrique Álvaro Brun

C.I.S. Victoria Kent

Servicios Sanitarios

C/ Juna de Vera, 10

28045 Madrid

E-mail: EnriqueAlvaro@saludalia.com 